\title{
Optimization of Drilling Parameters by Analysis of Formation Strength Properties with Utilization of Mechanical Specific Energy
}

\author{
Afshin Davarpanah1 ${ }^{*}$, Seyed Mohammad Mehdi Nassabeh², Behnam Mirshekari1 \\ ${ }^{1}$ Department of Petroleum Engineering, Science and Research Branch, Islamic Azad University, Tehran, Iran \\ ${ }^{2}$ Department of Petroleum Engineering, Faculty of Engineering and Technology, Omidiyeh Branch, Islamic Azad University, \\ Omidiyeh, Iran \\ Email: ^Afshin.Davarpanah@srbiau.ac.ir, Mehdi.nassabeh1@gmail.com, Mirshekari.Behnam@gmail.com
}

How to cite this paper: Davarpanah, A., Nassabeh, S.M.M. and Mirshekari, B. (2017) Optimization of Drilling Parameters by Analysis of Formation Strength Properties with Utilization of Mechanical Specific Energy. Open Journal of Geology, 7, 15901602.

https://doi.org/10.4236/ojg.2017.711107

Received: July 14, 2017

Accepted: November 6, 2017

Published: November 9, 2017

Copyright $\odot 2017$ by authors and Scientific Research Publishing Inc. This work is licensed under the Creative Commons Attribution International License (CC BY 4.0).

http://creativecommons.org/licenses/by/4.0/

\begin{abstract}
By increasing the daily needs of human energy, human manipulation of natural energy sources is expanded and encouraged the human society to developing science, knowledge and technology. Mechanical specific energy required energy for drilling the unit of formation volume. This parameter can be used for functional analysis of drilling, drilling bit optimization and investigating of instability has been made during drilling operations. This parameter can be used for decreasing of drilling costs by increasing drilling speed, optimized the useful life of the drilling bit and determine the right time to replace the drilling bit, and in some cases reduced to a minimum amount. In South Pars field in Iran, many wells have been drilled; however detailed statistics processes hadn't done for optimizing drilling parameters and their impact on mechanical specific energy. By results of these studies, we can analyze performance and drilling parameters such as weight on drilling bit, rotational speed, penetration rate, etc. In the most investigated cases, mechanical specific energy at the final period time of drilling on each wells has been increased gradually due to the speed movement reduction. Although by investigating middle formations in section of 12.25 inch, all existing wells on a platform in one of the phases of Iran's South Pars field are being studied, which contains formations such as Hith, Surmeh, Neyriz, Dashtak and Kangan. Studies were done in two parts. In the first part, the range of optimized drilling parameters that is increasing drilling speed and reducing the required amount of energy for drilling formation. This process by investigating mechanical specific energy and its relationship with uniaxial compressive strength in five studied formation have been presented. In the second part, correlations to predict the mechanical specific energy in this area by statistical methods by SPSS software, presented and reviewed. Then, by the most appropriate rela-
\end{abstract}


tionship, the most influential drilling parameters on mechanical specific energy have been set. However, for drilling the next wells in this area drilling parameters with the most priority influences on mechanical specific energy, proposed in the optimum range, will be recommended.

\section{Keywords}

Mechanical Specific Energy, Drilling Bit Penetration Rate, Compressive Strength, Statistical Methods

\section{Introduction}

Special mechanical energy is the required amount of energy for drilling the formation. This parameter is a function of drilling parameters such as weight on bit, rotational speed, torque, drilling penetration rate in the formation and the well diameter. Although if you used mud pumps, this parameter depended to proportion of speed to flow rate, the maximum rate of torque and maximum rate of pressure difference [1] [2] [3] [4] [5].

Mechanical specific energy for both relational concept and correlational was introduced in 1964 by Tail. Since this parameter used in the diagnosis of instability during drilling operations was valuable. Drilling engineers are always by applying different weights on bit, speed of rotation and mud flow rate within the range of normal operation [6] [7] [8] [9]. They try to minimize the amount of mechanical specific energy and maximize the penetration rate of bit on the formation. Drilling engineers always try to approach mechanical specific energy within current formation compressive strength. Unexpected changes in mechanical specific energy may indicate changes in the rock properties or drilling instabilities or both of them. In an ideal process, there is a relationship between input energy and an accurate compressive strength of a formation. However, this one by one relationship to compare with uniaxial compressive strength due to the effect of hydrostatic pressure of mud column on the depth, does not remain [10] [11] [12] [13].

By optimizing of mechanical specific energy, we can reduce unappreciable non-beneficial times and also drilling costs by using drilling optimization parameters for increasing beneficial life of bit, increasing penetration rate to maximum limit (without types of bit) and obtain optimum time for changing bit (when the performance of bit is lower than optimum limit) [14] [15] [16].

By accurate using of mechanical specific energy within analysis of this parameter, it can be diagnosed for slowing bit speed or damaged bits, choosing efficient bit for current formation, efficient rotation per minute and inhabitation of poor mud circulation. In Iran, despite drilling many wells in South Pars field, statistical research to optimize independent drilling parameters such as weight on bit, bit rotational speed, drilling speed and also its impact on each of the relevant parameters (mechanical specific energy) has not been done. Also due to 
high costs of hiring drilling rigs and limit use of some tools such as bit and borehole assembly that we have in Iran, this research is done for optimizing drilling parameters due to formation strength parameters by using mechanical specific energy. By investigating mechanical specific energy and its relationship with uniaxial compressive strength with increasing drilling speed, lengthen beneficial life of bit and borehole assembly, we can optimize costs that it is the main concern of petroleum companies [17] [18] [19].

\section{Working Procedure}

The principal of this research can be divided into two parts:

In the first part, the data are collected in the study area. Then which points that have higher drilling speed and also have lower mechanical specific energy and the ratio of mechanical specific energy to uniaxial compressive strength, in each well and formation to formation are separately recognized. Drilling parameters are collected at these point and with summarizing all of these data in all wells, for formations such as Hith, Surmeh, Neyriz, Dashtak and Kangan, range of optimum drilling parameters and so the amount of increasing penetration rate and decreasing mechanical specific energy will be determined. In the second part, firstly descriptive statistics, including the number of samples, the highest and lowest dispersion and its causes will examine. Then, in inferential statistics, there are some correlations for estimating mechanical specific energy by SPSS software in the studied area and their accuracy are investigated and optimum linear and non-linear correlation are determined. Then with the most appropriate correlation the most influential independent parameters on mechanical specific energy will be obtained. A total of first and second stages, for appropriate optimization of mechanical specific energy during drilling, efficient drilling parameters on mechanical specific energy(obtained in the second stage), in the optimal range, increasing drilling speed and reduce mechanical specific energy, for drilling other wells in this area (South Pars field in Iran) is recommended. More detailed statistical and inferential methods are further summarized.

\section{Case Study}

\subsection{Data Collection}

To collect and summarize all information related to wells 1 to 10 , in Table 1 . range of each independent parameters such as weight on bit, pressure difference, rotation per minute and flow rate, dependent drilling parameters, including penetration rate mechanical specific energy for section with 12.25 inch diameter that.

\subsubsection{Total Investigated Wells are Examined for Each Formation}

In studied area range of independent drilling parameters including the rotational velocity in the range of zero to 120 (RPM), weight on bit from zero to 223 (kIbs), mud flow rate from 200 to 9000 (psi), the pressure difference of 10 to 1270 (psi) 
Table 1. Optimization of drilling in the Hith Formation.

\begin{tabular}{|c|c|c|c|c|c|c|c|c|c|c|c|c|c|c|c|}
\hline Formation & well & Original Points & From & To & Drilled & ROP & $\mathrm{rpm}$ & $\begin{array}{c}\min \\
\text { WOB }\end{array}$ & $\begin{array}{c}\max \\
\text { WOB }\end{array}$ & Flow & $\Delta \mathrm{P}$ & $\begin{array}{c}\min \\
\mathrm{TQ}\end{array}$ & $\begin{array}{c}\max \\
\mathrm{TQ}\end{array}$ & MES & MES/UCS \\
\hline & & MSE (min) & 1853.2 & 1882.1 & 28.9 & 12.8444 & 40 & 15 & 20 & 843 & 340 & 10 & 13 & 69.551 & 0.46 \\
\hline & 1 & $\operatorname{rop}(\max )$ & 1930 & 1939 & 9 & 18 & 70 & 20 & 22 & 845 & 290 & 8 & 14 & 70.8595 & 0.469 \\
\hline & & MSE/UCS (min) & 1853.2 & 1882.1 & 28.9 & 12.8444 & 40 & 15 & 20 & 843 & 340 & 10 & 13 & 69.551 & 0.46 \\
\hline & & MSE (min) & 1840 & 1854 & 14.4 & 13.7 & 40 & 15 & 22 & 855 & 180 & 9 & 14 & 37.867 & 0.25 \\
\hline & 4 & $\operatorname{rop}(\max )$ & 1911 & 1939 & 28.3 & 24.8 & 120 & 19 & 24 & 855 & 300 & 5 & 13 & 81.858 & 0.542 \\
\hline & & MSE/UCS (min) & 1840 & 1854.4 & 14.4 & 13.7143 & 40 & 15 & 22 & 855 & 180 & 9 & 14 & 37.867 & 0.25 \\
\hline & & MSE (min) & 1980 & 1986 & 6 & 3.52941 & 90 & 12 & 15 & 795 & 280 & 0 & 0 & 0.00012 & $6.62 \mathrm{E}+07$ \\
\hline & 5 & $\operatorname{rop}(\max )$ & 2020 & 2030.9 & 10.9 & 16.0294 & 90 & 13 & 16 & 795 & 310 & 13 & 17 & 191.981 & 1.271 \\
\hline & & MSE/UCS (min) & 1980 & 1986 & 6 & 3.52941 & 90 & 12 & 15 & 795 & 280 & 0 & 0 & 0.00012 & $6.62 \mathrm{E}+07$ \\
\hline & & MSE (min) & 1877 & 1892 & 15 & 18.9873 & 40 & 18 & 24 & 900 & 300 & 7 & 11 & 33.0802 & 0.219 \\
\hline & 6 & $\operatorname{rop}(\max )$ & 1877 & 1892 & 15 & 18.9873 & 40 & 18 & 24 & 900 & 300 & 7 & 11 & 33.0802 & 0.219 \\
\hline & & MSE/UCS (min) & 1877 & 1892 & 15 & 18.9873 & 40 & 18 & 24 & 900 & 300 & 7 & 11 & 33.0802 & 0.219 \\
\hline & & MSE (min) & 1908 & 1911 & 3 & 9.09091 & 30 & 3 & 12 & 820 & 120 & 10 & 15 & 40.5985 & 0.268 \\
\hline \multirow[t]{14}{*}{ Hith } & 7 & $\operatorname{rop}(\max )$ & 1990.5 & 2019 & 28.5 & 21.1111 & 100 & 18 & 20 & 800 & 240 & 10 & 18 & 130.537 & 0.864 \\
\hline & & MSE/UCS (min) & 1908 & 1911 & 3 & 9.09091 & 30 & 3 & 12 & 820 & 120 & 10 & 15 & 40.5985 & 0.268 \\
\hline & & MSE (min) & 1766.5 & 1767.5 & 1 & 10 & 90 & 4 & 25 & 822 & 110 & 6 & 11 & 86.8285 & 0.575 \\
\hline & 9 & $\operatorname{rop}(\max )$ & 1835 & 1845 & 10 & 19.2308 & 105 & 19 & 21 & 835 & 250 & 5 & 13 & 126.76 & 0.839 \\
\hline & & MSE/UCS (min) & 1766.5 & 1767.5 & 1 & 10 & 90 & 4 & 25 & 822 & 110 & 6 & 11 & 86.8285 & 0.575 \\
\hline & & MSE (min) & 1752 & 1772 & 20 & 41.6667 & 50 & 15 & 18 & 797 & 220 & 10 & 12 & 25.8027 & 0.17 \\
\hline & 10 & $\operatorname{rop}(\max )$ & 1752 & 1772 & 20 & 41.6667 & 50 & 15 & 18 & 797 & 220 & 10 & 12 & 25.8027 & 0.17 \\
\hline & & MSE/UCS (min) & 1752 & 1772 & 20 & 41.6667 & 50 & 15 & 18 & 797 & 220 & 10 & 12 & 25.8027 & 0.17 \\
\hline & & MSE (min) & 1795 & 1809.8 & 14.8 & 13.2143 & 60 & 15 & 20 & 800 & 300 & 8 & 15 & 112.6 & 0.745 \\
\hline & 12 & $\operatorname{rop}(\max )$ & 1868 & 1895 & 27 & 26.7327 & 110 & 23 & 25 & 910 & 310 & 10 & 15 & 114.613 & 0.759 \\
\hline & & MSE/UCS (min) & 1795 & 1809.8 & 14.8 & 13.2143 & 60 & 15 & 20 & 800 & 300 & 8 & 15 & 112.6 & 0.745 \\
\hline & & MSE (min) & 1781.4 & 1799 & 17.6 & 9.16667 & 70 & 18 & 24 & 900 & 205 & 8 & 13 & 166.912 & 1.105 \\
\hline & 14 & $\operatorname{rop}(\max )$ & 1838.5 & 1867 & 28.5 & 11.5385 & 100 & 18 & 25 & 900 & 240 & 8 & 17 & 290.013 & 1.92 \\
\hline & & MSE/UCS (min) & 1781.4 & 1799 & 17.6 & 9.16667 & 70 & 18 & 24 & 900 & 205 & 8 & 13 & 166.912 & 1.105 \\
\hline
\end{tabular}

and of torque from 0 to 29 (kFT-Ib) is. This has led to a range of dependent drilling parameters include penetration rate of between 0.96 to $107.69(\mathrm{ft} / \mathrm{hr})$ and particularly mechanical specific energy between zero to 34292.5 (MPa).

\subsubsection{Identify the Optimum Points in Each of the Formations}

In the following tables, the areas with the highest drilling velocity, the lowest mechanical specific energy and the ratio of the lowest amount of mechanical specific energy to uniaxial compressive strength are the optimum points. by determining these points independent drilling parameters appropriate with those 
data that prepared these optimum points. This information is then presented in Tables 1-5.

\section{Statistical Method}

\subsection{Descriptive Statistics}

In this research to determine display characteristics of descriptive properties, a collection set data, the range of changes, minimum and maximum, average,

Table 2. Optimization of drilling in the Surmeh Formation.

\begin{tabular}{|c|c|c|c|c|c|c|c|c|c|c|c|c|c|c|c|}
\hline Formation & well & Original Points & From & To & Drilled & ROP & $\mathrm{rpm}$ & $\begin{array}{c}\min \\
\text { WOB }\end{array}$ & $\begin{array}{c}\max \\
\text { WOB }\end{array}$ & Flow & $\Delta \mathrm{P}$ & $\begin{array}{c}\min \\
\mathrm{TQ}\end{array}$ & $\begin{array}{c}\max \\
\text { TQ }\end{array}$ & MES & MES/UCS \\
\hline & & MSE (min) & 2566 & 2567 & 1 & 5.26 & 0 & 30 & 35 & 875 & 90 & 0 & 0 & 0.00028 & $2.82 \mathrm{E}-06$ \\
\hline & 1 & $\operatorname{rop}(\max )$ & 2069 & 2081.4 & 12.4 & 56.3636 & 115 & 18 & 24 & 875 & 440 & 7 & 13 & 51.2784 & 0.339592 \\
\hline & & MSE/UCS (min) & 2566 & 2567 & 1 & 5.26 & 0 & 30 & 35 & 875 & 90 & 0 & 0 & 0.00028 & $2.82 \mathrm{E}-06$ \\
\hline & & MSE (min) & 2397 & 2411 & 14 & 7.25389 & 0 & 1 & 10 & 800 & 100 & 0 & 0 & $4.67 \mathrm{E}-05$ & $5.1 \mathrm{E}-06$ \\
\hline & 4 & $\operatorname{rop}(\max )$ & 2194.5 & 2207 & 12.5 & 69.4444 & 120 & 20 & 25 & 900 & 300 & 7 & 17 & 45.2235 & 0.32 \\
\hline & & MSE/UCS (min) & 21638 & 2174.6 & 6.6 & 5.40698 & 0 & 5 & 8 & 900 & 180 & 0 & 0 & $5.52 \mathrm{E}-05$ & $5.1 \mathrm{E}-06$ \\
\hline & & MSE (min) & 2300 & 2304 & 4 & 9.09091 & 0 & 3 & 5 & 840 & 50 & 0 & 0 & $3.40 \mathrm{E}-05$ & $3.06 \mathrm{E}-07$ \\
\hline & 5 & $\operatorname{rop}(\max )$ & 2590 & 2600 & 10 & 43.4783 & 120 & 6 & 15 & 843 & 350 & 15 & 25 & 118.843 & 1.7 \\
\hline & & MSE/UCS (min) & 2094 & 2099.5 & 5.5 & 5.28846 & 0 & 4 & 5 & 795 & 50 & 0 & 0 & $3.82 \mathrm{E}-05$ & $3.06 \mathrm{E}-07$ \\
\hline & & MSE (min) & 1908 & 1914 & 6 & 5.40541 & & 10 & 20 & 900 & 140 & 0 & 0 & 0.00013 & $6.62 \mathrm{E}-07$ \\
\hline & 6 & $\operatorname{rop}(\max )$ & 2092 & 2092 & 12 & 70.5882 & 100 & 20 & 28 & 900 & 180 & 8 & 14 & 42.0923 & 0.298 \\
\hline & & MSE/UCS (min) & 1908 & 1914 & 6 & 5.40541 & & 10 & 20 & 900 & 140 & 0 & 0 & 0.00013 & $6.62 \mathrm{E}-07$ \\
\hline & & MSE (min) & 2981 & 2992.5 & 11.5 & 7.41936 & & 10 & 20 & 850 & 160 & 0 & 0 & 0.00013 & $1.02 \mathrm{E}-05$ \\
\hline & 7 & $\operatorname{rop}(\max )$ & 3005 & 3008 & 3 & 50 & 120 & 10 & 12 & 850 & 270 & 11 & 22 & 114.001 & 1.163 \\
\hline & & MSE/UCS (min) & 2822 & 2825.6 & 3.6 & 3.39623 & 0 & 5 & 25 & 800 & 150 & 0 & 0 & 0.00013 & $1.02 \mathrm{E}-05$ \\
\hline \multirow[t]{14}{*}{ Surmeh } & & MSE (min) & 2162.5 & 2165 & 2.55 & 3.89438 & 0 & 2 & 5 & 833 & 70 & 5 & 13 & $2.97 \mathrm{E}-05$ & $2.11 \mathrm{E}-07$ \\
\hline & 9 & $\operatorname{rop}(\max )$ & 2469 & 2475 & 6 & 35.2941 & 105 & 1 & 10 & 900 & 280 & 6 & 13 & 66.6123 & 0.662 \\
\hline & & MSE/UCS (min) & 2162.5 & 2165 & 2.55 & 3.89438 & 0 & 2 & 5 & 833 & 70 & 5 & 13 & $3 E-05$ & $2.11 \mathrm{E}-07$ \\
\hline & & MSE (min) & 2326 & 2330.4 & 4.4 & 3.89381 & 0 & 1 & 5 & 800 & 40 & 0 & 0 & $2.55 \mathrm{E}-05$ & $3.06 \mathrm{E}-07$ \\
\hline & & MSE/UCS (min) & 1954.5 & 1969.3 & 14.8 & 5.32374 & 0 & 0 & 5 & 800 & 100 & 0 & 0 & $2.6 \mathrm{E}-05$ & $1.99 \mathrm{E}-07$ \\
\hline & & MSE (min) & 2618.3 & 2621 & 2.7 & 10.3846 & & 5 & 10 & 950 & 100 & 14 & 22 & $6.37 \mathrm{E}-05$ & $5.56 \mathrm{E}-07$ \\
\hline & 11 & $\operatorname{rop}(\max )$ & 3050 & 3050.8 & 0.8 & 40 & & 80 & 80 & 898 & 70 & 0 & 0 & 0.00068 & $6.93 \mathrm{E}-05$ \\
\hline & & MSE/UCS (min) & 3050.8 & 2621 & -430 & 10.3846 & & 5 & 10 & 950 & 100 & 14 & 22 & $6.4 \mathrm{E}-05$ & $5.56 \mathrm{E}-07$ \\
\hline & & MSE (min) & 1981 & 2000.1 & 14.8 & 39.7917 & 110 & 19 & 23 & 905 & 350 & 11 & 17 & 97.3658 & 0.644 \\
\hline & 12 & $\operatorname{rop}(\max )$ & 2009.6 & 2038.5 & 27 & 46.6129 & 110 & 20 & 25 & 905 & 445 & 10 & 18 & 105.678 & 0.699 \\
\hline & & MSE/UCS(min) & 1981 & 2000.1 & 14.8 & 39.7917 & 110 & 19 & 23 & 905 & 350 & 11 & 17 & 97.3658 & 0.644 \\
\hline & & $\operatorname{MSE}(\min )$ & 1979 & 1982 & 17.6 & 42.8571 & 105 & 10 & 18 & 850 & 200 & 5 & 16 & 64.3015 & 0.425 \\
\hline & 14 & $\operatorname{rop}(\max )$ & 2066.5 & 2073 & 28.5 & 43.3333 & 105 & 12 & 20 & 850 & 375 & 6 & 19 & 145.324 & 1.03 \\
\hline & & MSE/UCS(min) & 1979 & 1982 & 17.6 & 42.8571 & 105 & 10 & 18 & 850 & 200 & 7 & 16 & 64.3015 & 0.425 \\
\hline
\end{tabular}


Table 3. Optimization of drilling in the Neyriz Formation.

\begin{tabular}{|c|c|c|c|c|c|c|c|c|c|c|c|c|c|c|c|}
\hline Formation & well & Original Points & From & To & Drilled & ROP & $\mathrm{rpm}$ & $\begin{array}{c}\min \\
\text { WOB }\end{array}$ & $\begin{array}{c}\max \\
\text { WOB }\end{array}$ & Flow & $\Delta \mathrm{P}$ & $\begin{array}{c}\min \\
\mathrm{TQ}\end{array}$ & $\begin{array}{c}\max \\
\mathrm{TQ}\end{array}$ & MES & MES/UCS \\
\hline & & MSE (min) & 2990 & 2998.7 & 8.7 & 28.0645 & 120 & 7 & 9 & 845 & 160 & 8 & 18 & 55.09 & $5.60 \mathrm{E}-01$ \\
\hline & 1 & $\operatorname{rop}(\max )$ & 2998.7 & 3004 & 5.3 & 31.1747 & 120 & 4 & 6 & 845 & 330 & 8 & 16 & 196.9897 & 0.927 \\
\hline & & MSE/UCS (min) & 2990 & 2998.7 & 8.7 & 28.0645 & 120 & 7 & 9 & 845 & 160 & 8 & 18 & 55.09 & 0.56 \\
\hline & & MSE (min) & 2927 & 2955.5 & 28.5 & 22.8 & 120 & 8 & 21 & 850 & 260 & 8 & 18 & $2.19 \mathrm{E}+02$ & 2.356 \\
\hline & 4 & $\operatorname{rop}(\max )$ & 2927 & 2955.5 & 28.5 & 22.8 & 120 & 8 & 21 & 850 & 260 & 8 & 18 & $2.19 \mathrm{E}+02$ & 2.356 \\
\hline & & MSE/UCS (min) & 2927 & 2955.5 & 28.5 & 22.8 & 120 & 8 & 21 & 850 & 260 & 8 & 18 & $2.19 \mathrm{E}+02$ & 2.356 \\
\hline & & MSE (min) & 3289.8 & 3295.3 & 5.8 & 14.5 & 120 & 8 & 11 & 922 & 130 & 15 & 22 & $1.53 \mathrm{E}+02$ & $1.56 \mathrm{E}+00$ \\
\hline & 5 & $\operatorname{rop}(\max )$ & 3295.3 & 3306.5 & 11.2 & 15.5556 & 120 & 6 & 10 & 922 & 200 & 17 & 22 & 231.89 & 2.366 \\
\hline & & MSE/UCS (min) & 3289.8 & 3295.3 & 5.8 & 14.5 & 120 & 8 & 11 & 922 & 130 & 15 & 22 & $1.53 \mathrm{E}+02$ & $1.56 \mathrm{E}+00$ \\
\hline & & MSE (min) & 2789.8 & 2818 & 28.2 & 17.7359 & 120 & 15 & 18 & 900 & 325 & 8 & 19 & 96.71303 & $4.58 \mathrm{E}+00$ \\
\hline & 6 & $\operatorname{rop}(\max )$ & 2789.8 & 2818 & 28.2 & 17.7359 & 120 & 15 & 18 & 900 & 325 & 8 & 19 & 96.71303 & 4.577 \\
\hline & & MSE/UCS (min) & 2789.8 & 2818 & 28.2 & 17.7359 & 120 & 15 & 18 & 900 & 325 & 8 & 19 & 96.71303 & 4.577 \\
\hline & & MSE (min) & 3033.6 & 3061.3 & 27.7 & 15.0544 & 120 & 15 & 25 & 750 & 300 & 10 & 21 & 395.203 & 2.224 \\
\hline & 7 & $\operatorname{rop}(\max )$ & 3033.6 & 3061.3 & 27.7 & 15.0544 & 120 & 15 & 25 & 750 & 300 & 10 & 21 & 395.203 & 2.224 \\
\hline & & MSE/UCS (min) & 3033.6 & 3061.3 & 27.7 & 15.0544 & 120 & 15 & 25 & 750 & 300 & 10 & 21 & 395.203 & 2.224 \\
\hline \multirow[t]{15}{*}{ NEYRIZ } & & MSE (min) & 2773 & 2788 & 15 & 20.548 & 120 & 10 & 20 & 895 & 350 & 6 & 13 & $1.77 \mathrm{E}+02$ & $1.63 \mathrm{E}+00$ \\
\hline & 9 & $\operatorname{rop}(\max )$ & 2773 & 2788 & 15 & 20.548 & 120 & 10 & 20 & 895 & 350 & 6 & 13 & 177.4622 & 1.625 \\
\hline & & MSE/UCS (min) & 2773 & 2788 & 15 & 20.548 & 120 & 10 & 20 & 895 & 350 & 6 & 13 & 177.4622 & 1.625 \\
\hline & & MSE (min) & 2689.9 & 2718.7 & 28.8 & 28.2353 & 60 & 9 & 22 & 800 & 330 & 5 & 17 & $6.33 \mathrm{E}+01$ & $9.35 \mathrm{E}-01$ \\
\hline & 10 & $\operatorname{rop}(\max )$ & 2689.9 & 2718.7 & 28.8 & 28.2353 & 60 & 9 & 22 & 800 & 330 & 5 & 17 & 63.26608 & 0.935 \\
\hline & & MSE/UCS (min) & 2689.9 & 2718.7 & 28.8 & 28.2353 & 60 & 9 & 22 & 800 & 330 & 5 & 17 & 63.26608 & $9.35 \mathrm{E}-01$ \\
\hline & & MSE (min) & 3430.4 & 3459 & 28.6 & 16.7252 & 100 & 5 & 10 & 950 & 160 & 14 & 26 & $3.82 \mathrm{E}+02$ & $2.66 \mathrm{E}+00$ \\
\hline & 11 & $\operatorname{rop}(\max )$ & 3430.4 & 3459 & 28.6 & 16.7252 & 100 & 5 & 10 & 950 & 160 & 14 & 26 & 382.498 & 2.657 \\
\hline & & MSE/UCS (min) & 3430.4 & 3459 & 28.6 & 16.7252 & 100 & 5 & 10 & 950 & 160 & 14 & 26 & 382.498 & 2.657 \\
\hline & & MSE (min) & 3072.7 & 3101 & 28.3 & 13.4123 & 120 & 15 & 23 & 905 & 220 & 10 & 20 & 429.2771 & 2.536 \\
\hline & 12 & $\operatorname{rop}(\max )$ & 3072.7 & 3101 & 28.3 & 13.4123 & 120 & 15 & 23 & 905 & 220 & 10 & 20 & 429.7771 & 2.536 \\
\hline & & MSE/UCS (min) & 3072.7 & 3101 & 28.3 & 13.4123 & 120 & 15 & 23 & 905 & 220 & 10 & 20 & 429.2771 & 2.536 \\
\hline & & MSE (min) & 2785.3 & 2814 & 28.7 & 18.9934 & 120 & 10 & 15 & 880 & 170 & 9 & 16 & 143.1483 & 1.409 \\
\hline & 14 & $\operatorname{rop}(\max )$ & 2785.3 & 2814 & 28.7 & 18.9934 & 120 & 10 & 15 & 880 & 170 & 9 & 16 & 143.1483 & 1.409 \\
\hline & & MSE/UCS $(\min )$ & 2785.3 & 2814 & 28.7 & 18.9934 & 120 & 10 & 15 & 880 & 170 & 9 & 16 & 143.1483 & 1.409 \\
\hline
\end{tabular}

offset scale and gear ratio of changes are provided in the Tables 1-8 that the purpose of this investigate to affect any of the existing parameters on mechanical specific energy. That's why parameters ( $T Q, \Delta P, Q, W O B, N, R O P$ and drilled) become independent variables. Changes in these parameters will change in the MSE as a dependent parameter. 
Table 4. Optimization of drilling in the Dashtak Formation.

\begin{tabular}{|c|c|c|c|c|c|c|c|c|c|c|c|c|c|c|c|}
\hline Formation & well & Original Points & From & To & Drilled & ROP & $\mathrm{rpm}$ & $\begin{array}{c}\min \\
\text { WOB }\end{array}$ & $\begin{array}{c}\max \\
\text { WOB }\end{array}$ & Flow & $\Delta \mathrm{P}$ & $\begin{array}{c}\min \\
\mathrm{TQ}\end{array}$ & $\begin{array}{c}\max \\
\mathrm{TQ}\end{array}$ & MES & MES/UCS \\
\hline & & MSE (min) & 3314.5 & 3315.5 & 1 & 3.44828 & 115 & 20 & 30 & 857 & 10 & 11 & 13 & 10.91976 & $7.50 \mathrm{E}-01$ \\
\hline & 1 & $\operatorname{rop}(\max )$ & 3004 & 3007.9 & 3.9 & 27.8571 & 120 & 1 & 2 & 845 & 260 & 8 & 16 & 45.1 .3396 & 0.818 \\
\hline & & MSE/UCS (min) & 3314.5 & 3315.5 & 1 & 3.44828 & 115 & 20 & 30 & 857 & 10 & 11 & 13 & 10.91976 & 0.75 \\
\hline & & MSE (min) & 2997 & 3003.7 & 6.7 & 3.52632 & 0 & 10 & 22 & 850 & 160 & 0 & 0 & $1.36 \mathrm{E}-04$ & $1.02 \mathrm{E}-06$ \\
\hline & 4 & $\operatorname{rop}(\max )$ & 2958 & 2960 & 2 & 25 & 120 & 10 & 18 & 850 & 150 & 8 & 18 & $1.03 \mathrm{E}+02$ & 1.0911 \\
\hline & & MSE/UCS (min) & 2997 & 3003.7 & 6.7 & 3.52632 & 0 & 10 & 22 & 850 & 160 & 0 & 0 & $1.36 \mathrm{E}-04$ & $1.02 \mathrm{E}-06$ \\
\hline & & MSE (min) & 3323.4 & 3334 & 10.6 & 14.3243 & 120 & 10 & 15 & 922 & 180 & 15 & 24 & $2.67 \mathrm{E}+02$ & $1.77 \mathrm{E}+00$ \\
\hline & 5 & $\operatorname{rop}(\max )$ & 3493.4 & 3514 & 20.2 & 19.2381 & 120 & 20 & 25 & 920 & 240 & 21 & 24 & 259.6177 & 1.643 \\
\hline & & MSE/UCS (min) & 3514 & 3522.1 & 8.1 & 16.5306 & 120 & 18 & 23 & 920 & 195 & 20 & 24 & $2.40 \mathrm{E}+02$ & $1.52 \mathrm{E}+00$ \\
\hline & & MSE (min) & 2826 & 2827 & 1 & 6.66667 & 100 & 2 & 6 & 900 & 60 & 9 & 12 & 94.46068 & $7.37 \mathrm{E}-01$ \\
\hline & 6 & $\operatorname{rop}(\max )$ & 2827 & 2830 & 3 & 13.6364 & 100 & 9 & 12 & 900 & 240 & 9 & 26 & 307.8718 & 2.405 \\
\hline & & MSE/UCS (min) & 2826 & 2827 & 1 & 6.66667 & 100 & 2 & 6 & 900 & 60 & 9 & 12 & 94.46068 & 0.737 \\
\hline & & MSE (min) & 3443 & 3444 & 1 & 8.33333 & 80 & 5 & 8 & 620 & 85 & 13 & 19 & 160.62 & 1.46 \\
\hline & 7 & $\operatorname{rop}(\max )$ & 3374 & 3388 & 14 & 21.2121 & 120 & 10 & 15 & 800 & 340 & 12 & 26 & 2982.986 & 2.838 \\
\hline \multirow{16}{*}{ Dashtak } & & MSE/UCS (min) & 3443 & 3444 & 1 & 8.33333 & 80 & 5 & 8 & 620 & 85 & 13 & 19 & 160.62 & 1.46 \\
\hline & & MSE (min) & 3164 & 3165 & 1 & 12.5 & 80 & 15 & 20 & 835 & 240 & 6 & 12 & $1.34 \mathrm{E}+02$ & $1.41 \mathrm{E}+00$ \\
\hline & 9 & $\operatorname{rop}(\max )$ & 2801 & 2807.5 & 6.5 & 17.5676 & 105 & 14 & 16 & 900 & 370 & 7 & 11 & 163.24 & 1.275 \\
\hline & & MSE/UCS (min) & 2994 & 2995 & 1 & 3.7037 & 80 & 1 & 5 & 800 & 100 & 7 & 14 & 137.397 & 0.869 \\
\hline & & MSE (min) & 2747 & 2775.7 & 28.7 & 19.7931 & 60 & 18 & 22 & 800 & 320 & 7 & 12 & $7.86 \mathrm{E}+01$ & $6.14 \mathrm{E}-01$ \\
\hline & 10 & $\operatorname{rop}(\max )$ & 2718.7 & 2747 & 28.3 & 21.4394 & 60 & 15 & 22 & 800 & 320 & 8 & 16 & 91.66605 & 0.935 \\
\hline & & MSE/UCS(min) & 2747 & 2775.7 & 28.7 & 19.7931 & 60 & 18 & 22 & 800 & 320 & 7 & 12 & 78.60491 & $6.14 \mathrm{E}-01$ \\
\hline & & MSE(min) & 3587.5 & 3587.7 & 0.2 & 6.66667 & 10 & 1 & 1 & 200 & 10 & 20 & 20 & $3.20 \mathrm{E}+00$ & $2.40 \mathrm{E}-02$ \\
\hline & 11 & $\operatorname{rop}(\max )$ & 3459 & 3482.4 & 23.4 & 15.1948 & 100 & 10 & 25 & 950 & 222 & 14 & 26 & 311.5558 & 4.058 \\
\hline & & MSE/UCS (min) & 3587.9 & 3587.7 & 0.2 & 6.66667 & 10 & 1 & 1 & 200 & 10 & 20 & 20 & 3.198676 & 0.024 \\
\hline & & MSE (min) & 3427 & 3427.5 & 0.5 & 3.57143 & 50 & 10 & 10 & 905 & 30 & 17 & 17 & 83.04912 & 0.525 \\
\hline & 12 & $\operatorname{rop}(\max )$ & 3441.1 & 3469.5 & 28.4 & 16.7929 & 100 & 20 & 25 & 905 & 260 & 17 & 20 & 333.1624 & 2.289 \\
\hline & & MSE/UCS (min) & 3427 & 3427.5 & 0.5 & 3.57143 & 50 & 10 & 10 & 905 & 30 & 17 & 17 & 83.04912 & 0.525 \\
\hline & & $\operatorname{MSE}(\min )$ & 3199 & 3213 & 14 & 107.692 & 100 & 20 & 25 & 880 & 150 & 10 & 15 & 23.20481 & 0.174 \\
\hline & 14 & $\operatorname{rop}(\max )$ & 3199 & 3213 & 14 & 107.692 & 100 & 20 & 25 & 880 & 150 & 10 & 15 & 23.20481 & 0.174 \\
\hline & & MSE/UCS(min) & 3199 & 3213 & 14 & 107.692 & 100 & 20 & 25 & 880 & 150 & 10 & 15 & 23.20481 & 0.174 \\
\hline
\end{tabular}

The number of collection samples are 1485 that show the importance of derived correlations from the investigated formations in different wells. The range of changes and the offset scale, is important indicators for measuring volume dispersion amount one variable a. Average is the most important and easiest central index data. Using the average it can be compared many number of variables together. Gear ratio of the changes obtained from divided offset scale of 
Table 5. Optimization of drilling in the Kangan Formation.

\begin{tabular}{|c|c|c|c|c|c|c|c|c|c|c|c|c|c|c|c|}
\hline Formation & well & Original Points & From & To & Drilled & ROP & $\mathrm{rpm}$ & $\begin{array}{c}\min \\
\text { WOB }\end{array}$ & $\begin{array}{c}\max \\
\text { WOB }\end{array}$ & Flow & $\Delta \mathrm{P}$ & $\begin{array}{c}\min \\
\text { TQ }\end{array}$ & $\begin{array}{c}\max \\
\text { TQ }\end{array}$ & MES & MES/UCS \\
\hline & & MSE (min) & 3750 & 3758 & 8 & 10.3896 & 115 & 25 & 28 & 846 & 290 & 13 & 15 & 429.0907 & $3.90 \mathrm{E}+00$ \\
\hline & 1 & $\operatorname{rop}(\max )$ & 3758 & 3766 & 8 & 10.8108 & 115 & 25 & 28 & 846 & 335 & 13 & 15 & 476.3617 & 2.977 \\
\hline & & MSE/UCS (min) & 3766 & 3768 & 2 & 10 & 105 & 25 & 28 & 846 & 190 & 13 & 15 & 470.2043 & 2.938 \\
\hline & 5 & $\operatorname{rop}(\max )$ & 4121.6 & 4127 & 5.4 & 5 & 120 & 18 & 21 & 800 & 150 & 20 & 25 & 1439.4 & 8.996 \\
\hline & & MSE/UCS (min) & 4121.6 & 4127 & 5.4 & 5 & 120 & 18 & 21 & 800 & 150 & 20 & 25 & 1439.4 & $9.00 \mathrm{E}+00$ \\
\hline & & MSE (min) & 3441 & 3442 & 1 & 1.96078 & 110 & 30 & 33 & 900 & 80 & 9 & 11 & 717.7812 & $6.53 \mathrm{E}+00$ \\
\hline \multirow[t]{8}{*}{ Kangan } & 6 & $\operatorname{rop}(\max )$ & 3460 & 3462.5 & 2.5 & 2.23214 & 110 & 30 & 33 & 900 & 120 & 9 & 14 & 1673.307 & 10.458 \\
\hline & & MSE/UCS (min) & 3441 & 3442 & 1 & 1.96078 & 110 & 30 & 33 & 900 & 80 & 9 & 11 & 717.7812 & 6.525 \\
\hline & & MSE (min) & 4253.8 & 4261 & 7.2 & 2.75862 & 110 & 20 & 30 & 850 & 110 & 25 & 29 & $2.53 \mathrm{E}+03$ & $2.30 \mathrm{E}+01$ \\
\hline & 11 & $\operatorname{rop}(\max )$ & 4253.8 & 4261 & 7.2 & 2.75862 & 110 & 20 & 30 & 850 & 110 & 25 & 29 & 2525.428 & 22.958 \\
\hline & & MSE/UCS (min) & 4253.8 & 4261 & 7.2 & 2.75862 & 110 & 20 & 30 & 850 & 110 & 25 & 29 & 2525.428 & 22.958 \\
\hline & & MSE (min) & 3526 & 3550 & 24 & 6.4 & 120 & 24 & 28 & 875 & 160 & 11 & 16 & 809.6629 & 7.36 \\
\hline & 14 & $\operatorname{rop}(\max )$ & 3526 & 3550 & 24 & 6.4 & 120 & 24 & 28 & 875 & 160 & 11 & 16 & 809.6629 & 7.36 \\
\hline & & MSE/UCS (min) & 3526 & 3550 & 24 & 6.4 & 120 & 24 & 28 & 875 & 160 & 11 & 16 & 809.6629 & 7.36 \\
\hline
\end{tabular}

Table 6. Optimum range of independent drilling parameters in total investigated wells.

\begin{tabular}{|c|c|c|c|c|c|c|c|c|c|c|c|c|c|c|c|}
\hline \multirow[b]{2}{*}{ Formation } & \multicolumn{3}{|c|}{$\mathrm{N}(\mathrm{RPM})$} & \multicolumn{3}{|c|}{ WOB (kIbs) } & \multicolumn{3}{|c|}{ Flow (gal/min) } & \multicolumn{3}{|c|}{$\Delta \mathrm{P}(\mathrm{psi})$} & \multicolumn{3}{|c|}{ TQ (KFT-Ib) } \\
\hline & $\min$ & $\max$ & $\begin{array}{l}\text { Average } \\
\text { (Around) }\end{array}$ & $\min$ & $\max$ & $\begin{array}{l}\text { Average } \\
\text { (Around) }\end{array}$ & $\min$ & $\max$ & $\begin{array}{l}\text { Average } \\
\text { (Around) }\end{array}$ & $\min$ & $\max$ & $\begin{array}{l}\text { Average } \\
\text { (Around) }\end{array}$ & $\min$ & $\max$ & $\begin{array}{l}\text { Average } \\
\text { (Around) }\end{array}$ \\
\hline Hith & 30 & 120 & 66.85 & 3 & 25 & 17.55 & 795 & 910 & 840.77 & 110 & 340 & 243.33 & 0 & 18 & 10.22 \\
\hline Surmeh & 0 & 120 & 77.5 & 0 & 80 & 35.75 & 795 & 950 & 864.4 & 40 & 445 & 195.33 & 0 & 25 & 7.43 \\
\hline Neyriz & 60 & 120 & 111 & 4 & 25 & 33.65 & 750 & 950 & 869.7 & 130 & 350 & 248.5 & 5 & 26 & 14.15 \\
\hline Dashtak & 0 & 120 & 81.5 & 1 & 30 & 33.63 & 200 & 950 & 808.36 & 10 & 370 & 163.9 & 0 & 26 & 13.61 \\
\hline Kangan & 105 & 120 & 114.33 & 18 & 33 & 25.7 & 800 & 900 & 854.2 & 80 & 335 & 157 & 9 & 29 & 17.5 \\
\hline
\end{tabular}

Table 7. Optimum range of dependent drilling parameters in total investigated wells.

\begin{tabular}{|c|c|c|c|c|c|c|c|c|c|}
\hline \multirow[b]{2}{*}{ Formation } & \multicolumn{3}{|c|}{$\mathrm{ROP}(\mathrm{ft} / \mathrm{hr})$} & \multicolumn{3}{|c|}{ MSE(Mpa) } & \multicolumn{3}{|c|}{ MSE/UCS } \\
\hline & $\min$ & $\max$ & $\begin{array}{l}\text { Average } \\
\text { (Around) }\end{array}$ & $\min$ & $\max$ & $\begin{array}{l}\text { Average } \\
\text { (Around) }\end{array}$ & $\min$ & $\max$ & $\begin{array}{l}\text { Average } \\
\text { (Around) }\end{array}$ \\
\hline Hith & 3.53 & 41.66 & 17.13 & 0.000758423 & 1999.549 & 578.7459 & $4.57 \mathrm{E}-06$ & 13.23793 & 3.73695845 \\
\hline Surmeh & 3.4 & 70.59 & 25.16 & $1.75 \mathrm{E}-04$ & 1001.946 & 243.1781 & $1.37 \mathrm{E}-06$ & 11.72109 & 2.11669048 \\
\hline Neyriz & 13.41 & 31.14 & 19.745 & 379.8321786 & 2959.712 & 1508.78 & 3.861064 & 31.5573 & 14.3410951 \\
\hline Dashtak & 3.44 & 107.69 & 21.73 & 0.000930792 & 2297.071 & 816.0635 & $7.03 \mathrm{E}-06$ & 27.97893 & 7.27396893 \\
\hline Kangan & 1.96 & 10.81 & 5.32 & 2598.471401 & 17412.16 & 8645.06 & 20.2568 & 158.2898 & 69.4991534 \\
\hline
\end{tabular}


Table 8. Descriptive characteristics of data collection that are used.

\begin{tabular}{cccccccc}
\hline Parameter & Unit & Range of changes & Minimum & Maximum & average & Offset scale & Ratio of changes \\
\hline Drilled & $\mathrm{m}$ & 29.6 & 0 & 29.6 & 10.36 & 9.033 & 0.872 \\
ROP & $\mathrm{ft} / \mathrm{hr}$ & 38.49 & 1.3 & 39.79 & 11.75 & 8.31 & 0.707 \\
$\mathrm{~N}$ & $\mathrm{RPM}$ & 110 & 20 & 130 & 103.89 & 19.938 & 0.192 \\
$\mathrm{WOB}$ & $\mathrm{Klbs}$ & 32.5 & 1 & 33.5 & 18.96 & 7.381 & 0.389 \\
$\mathrm{Q}$ & $\mathrm{GPM}$ & 300 & 650 & 950 & 866.75 & 49.171 & 0.057 \\
$\mathrm{SP}$ & $\mathrm{psi}$ & 510 & 10 & 520 & 225.49 & 81.034 & 0.359 \\
$\mathrm{TQ}$ & $\mathrm{Kft.lbs}$ & 20 & 7 & 27 & 15.04 & 4.694 & 0.312 \\
$\mathrm{MSE}$ & $\mathrm{ksi}$ & 3366.97 & 19.62 & 3386.59 & 494.53 & 468.977 & 0.948 \\
\hline
\end{tabular}

one variable on average, relative index for comparing different variables, whatever the gear ratio of the changes to be more, variable distribution will be more. Mechanical Specific energy has the biggest range of changes, the offset scale and gear ratio of changes. High dispersion of this variable, due to the different resistor properties in different lithology. After mechanical specific energy, pressure difference has the highest scope of the changes and the offset scale, low pressure difference relevant to low special weight and high pressure difference represents high special weight of cuttings in that part. The least range of changes and the offset scale related to the torque and the lowest gear ratio of changes related to the mud flow rate that is revealed parameters were close and less distribution.

The histogram is a method to display the distribution of data in different quantity categories. The range of parameters related to this region in addition to the table descriptive characteristics specified in Figure 1. Proposal relations related to this ranges and if we use these equations for outside parameters of the range it needs more careful nesses and more caution. Furthermore, Normal curve and histogram of drilling parameters are being clearly plotted in Figure 2.

\subsection{Inferential Statistics}

As we know, this statistical method can refer to regression method. In the inferential statistics, linear correlations and non-linear correlations are evaluated.

\subsection{Wrap up}

In this case firstly descriptive statistics that includes number of samples, the most and the least dispersion and their reasons are review. For the studied area, investigated descriptive characteristics shows the range of the changes, the offset scale and high gear ranges of mechanical specific energy, pressure differences and true vertical depth that shows more dispersion on this variables. Low amount of these parameters such as torque, rotational speed and mud flow rate, revealed low dispersion for this parameters.

Then, in inferential statistics there were correlations to predict mechanical specific energy in the studied area by using statistical methods with SPSS 


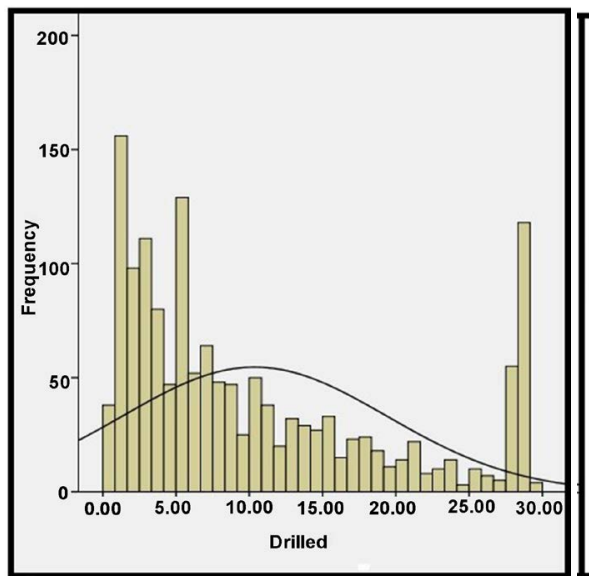

(a)

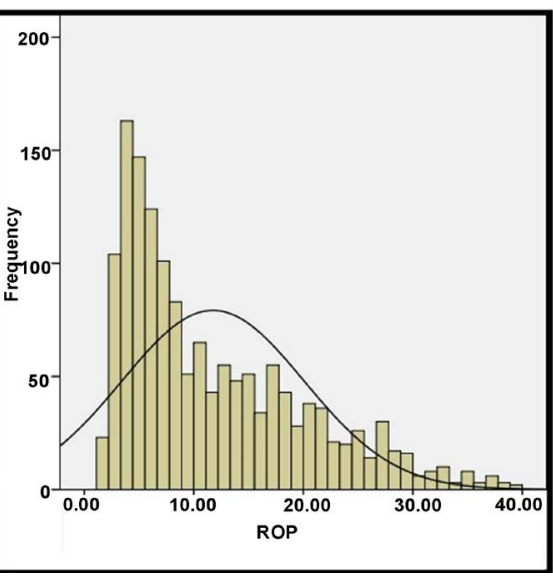

(b)

Figure 1. Normal curve and histogram of drilling parameters. (a) Drilled; (b) ROP.

software was presented. The most appropriate Linear and non-linear correlations were investigated. By investigating suggested correlations in the studied field and compare their accuracy, the following results were obtained:

In the proposed linear equations, linear correlation without intercept, with number of test 383/946, correlation coefficients 0/904 and coefficients of determination $0 / 818$, is superior and more reliable than linear correlation with intercept, the statistic test $344 / 567$, correlation coefficients $0 / 788$ and coefficient of determination is $0 / 62$.

In comparison of non-linear correlation, proposed non-linear correlation with a coefficient of determination $0 / 775$, the two proposed non-linear correlations to state that we didn't use the mud pump in the well coefficient of determination were $0 / 819$ and $0 / 813$. When we use the mud pump in the well coefficient of determination were $0 / 837$ and $0 / 832$ that compared to other non-linear correlations were higher. This represents a further connection between the parameters and higher accuracy, especially in mechanical specific energy correlation with other independent drilling parameters into two correlations.

\section{Conclusions}

1) To use the results of this study, it should be considered that the range of parameters provided, in addition to the descriptive specification table, in normal curve and drilling histogram parameters are specified. As well as from a variety of statistical methods, SPSS software was used in this research. Several proposed correlations for mechanical specific energy were done by this software.

2) It is recommended that the neural network application software must be designed to determine with serial changes in drilling parameters, the most appropriate model for achieving the optimal combination of mechanical specific energy.

3) As noted above, the mechanical specific energy can help for checking drilling performance (selecting and optimizing drilling parameters), checking the performance of bit (bit design with more efficiency) and the instability of 


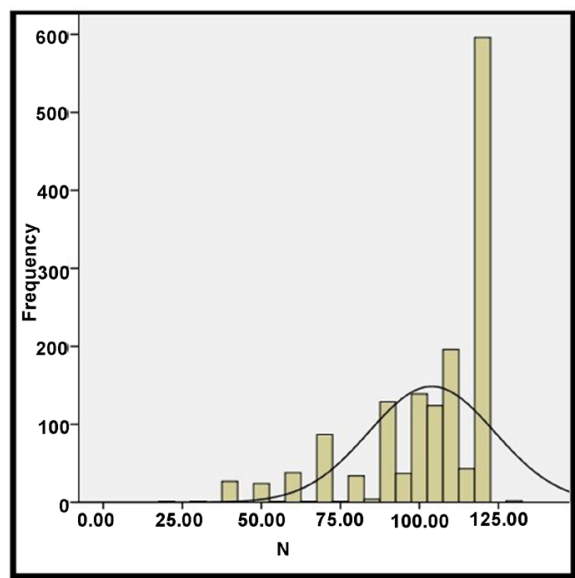

(a)

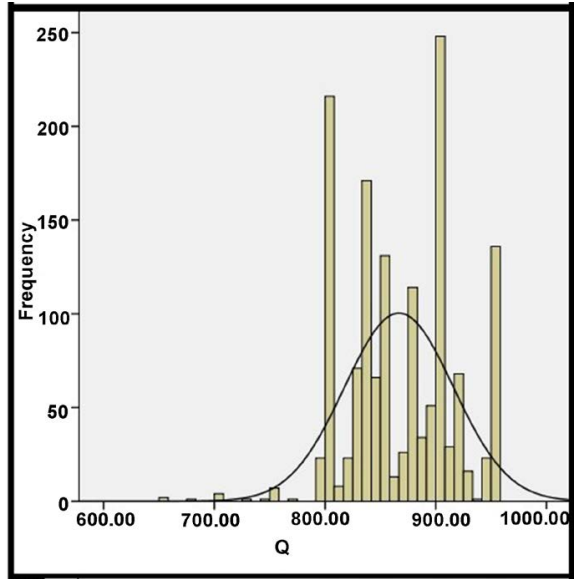

(c)

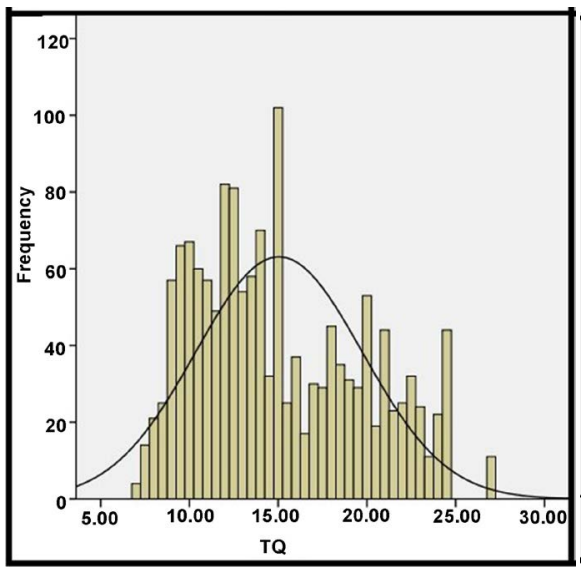

(d)

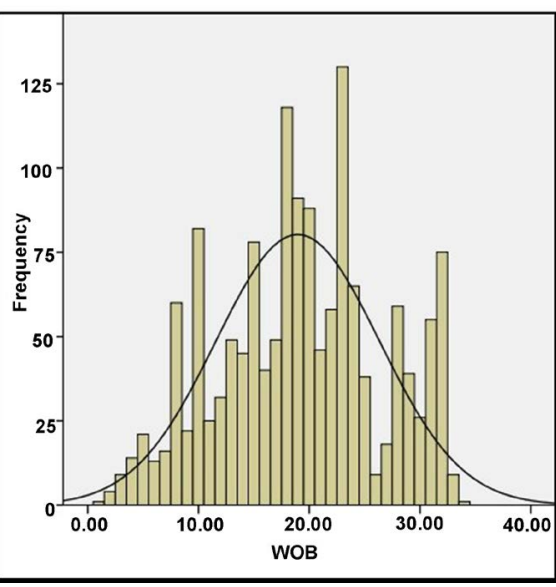

(b)

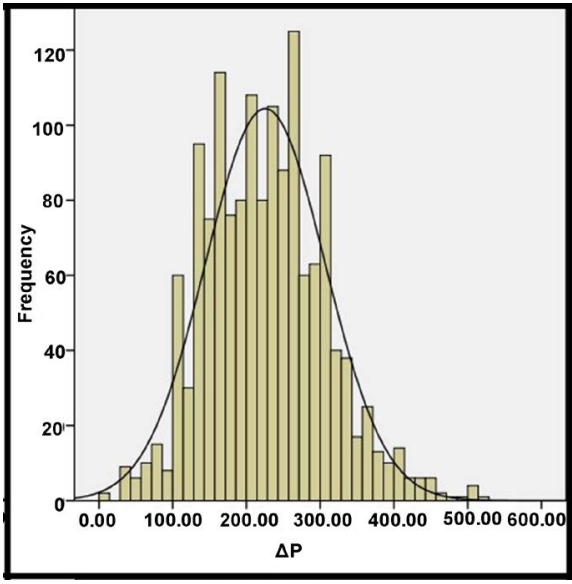

(d)

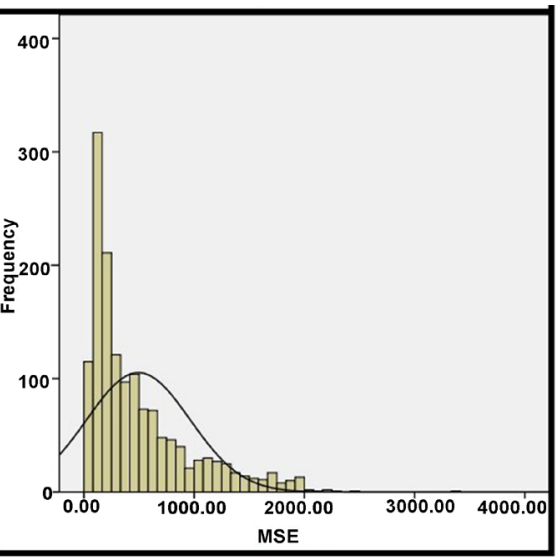

(f)

Figure 2. Normal curve and histogram of drilling parameters. (a) N; (b) WOB; (c) Q; (d) $\triangle \mathrm{P}$; (e) TQ; (f) MSE.

drilling operations. Although appropriate statistical processed for optimizing drilling parameters and their effect on mechanical specific energy on the studied area hadn't been done and also mechanical specific energy have an important role in reducing costs. In this research by using mechanical specific energy, we 
can analyze and optimize drilling parameters on the part of Iran's South Pars field. As a result of this research, it recommended that by using mechanical specific energy to investigate drilling instabilities during operation (which can include bit plunging and BHA in the mud, vibrations, improper cleaning of wells, etc.) and checking the bit performance in the area. By selecting the appropriate type of bit (fixed cutter, PCD, etc.), appropriately designed of bit (number of blades, size and density of cutters, number and size of nozzles) and taking cutting depth, adjacent pressure and etc., must be increased drilling speed and minimize the damages to the bit.

\section{References}

[1] Amadi, W.K. and Iyalla, I. (2012) Application of Mechanical Specific Energy Techniques in Reducing Drilling Cost in Deepwater Development. Paper SPE 156370 MS Presented at the SPE Deep Water Drilling and Completion Conference, Galveston.

[2] Azike-Akubue, V., Barton, S., Gee, R. and Burnett, T. (2012) SPE, National Oilwell Varco Agitation Tools Enable Significant Reduction in Mechanical Specificenergy. Paper SPE 158240-MS Presented at the SPE Asia Pacific Oil and Gas Conference and Exhibition, Perth.

[3] Bradford, J., Ferrari, LA., Rickabaugh, C., Rothe, M. and Tipton, B. (2012) Hybrid Drill Bitcombining Fixed-Cutter and Roller-Cone Elements Improves Drilling Performance in Marcellus Shale Surface Interval. Paper SPE 154831 Presented at the Americas Unconventional Resources Conference, Pennsylvania.

[4] Caicedo, H., Calhoun, W. and Ewy, R. (2005) Unique ROP Predictor Using Bitspecific Coefficient of Sliding Friction and Mechanical Efficiency as a Function of Confined Compressive Strength Impacts Drilling Performance. Paper SPE 92576 Presented at 2005 SPE Drilling Conference, Amsterdam.

[5] Dupriest, F., Mobil, E.L. and Koederitz, W. (2005) Maximizing Drill Rates with Real-Time Surveillance of Mechanical Specific Energy. Paper SPE/IADC 92194 Presented at the SPE/IADC Drilling Conference, Amsterdam.

[6] Hammoutene, C. and Bits, S. (2012) FEA Modelled MSE/UCS Values Optimize PDC Design for Entire Hole Section, North Africa Technical Conference and Exhibition, Cairo, 20-22 February 2012, SPE-149372-MS.

[7] Kim, S.Y. (2008) Comparison of the Decision Tree, Artificial Neural Network, Andlinear Regression Method Based on the Number and Types of Independent Variables and Sample Size. Expert System with Application, 34, 1227-1234. https://doi.org/10.1016/j.eswa.2006.12.017

[8] Mensa-Wilmot, G., James, B., Bits, S., Aggarwal, L. and Van Luu, H. (2006) Gage Design-Effects of Gage Pad Length, Geometry, and Activity (Side Cutting) on PDC Bit Stability, Steer Ability, and Borehole Quality in Rotary Steerable. IADC/SPE Drilling Conference, Miami, 21-23 February 2006, SPE-98931-MS.

[9] Mohan, K., Adil, F. and Halliburton, S.R. (2009) Tracking Drilling Efficiency Using Hydro-Mechanical Specific Energy. SPE/IADC Drilling Conference and Exhibition, Amsterdam, 17-19 March 2009, SPE-119421-MS.

[10] NIST (2010) Structural Robustness Large-Scale Testing. https://www.nist.gov/el/mssd/structural-robustness-large-scale-testing

[11] Rajabov, V. and Miska, S. (2012) The Effects of Back-Rake and Side-Rake Angles on 
Mechanical Specific Energy of Single PDC Cutters with Selected Rocks at Varying Depth of Cuts and Confining Pressures. IADC/SPE Drilling Conference and Exhibition, San Diego, 6-8 March 2012, SPE-151406-MS.

[12] Remmert, S.M., Witt, J.W. and Dupriest, F.E. (2007) Implementation of ROP Management Process in Qatar North Field. SPE/IADC Drilling Conference, Amsterdam, 20-22 February 2007, SPE-105521-MS.

[13] Guerrero, C. (2007) SPE Drilling Studies Group, Drilling Engineer, Drilling Solutions Team. Drilling Optimization with Mechanical Specific Energy.

[14] Waughman, R., Kenner, J. and Moore, R. (2002) Real-Time Specific Energy Monitoring Reveals Drilling Inefficiency and Enhances the Understanding of When to Pull Worn PDC Bits. IADC/SPE Drilling Conference, Dallas, 26-28 February 2002, SPE-74520-MS.

[15] MSE (2010) A Valuable Trending Tool for Drillers and Engineers.

[16] Davarpanah, A., Zarei, M. and Mehdi-Nassabeh, S.M. (2016) Assessment of Mechanical Specific Energy Aimed at Improving Drilling Inefficiencies and Minimize Wellbore Instability. Journal of Petroleum and Environmental Biotechnology, 7, 309.

[17] Al-Qahtani, M. and Zillur, R. (2001) A Mathematical Algorithm for Modeling Geomechanical Rock Properties of the Khuff and Pre-Khuff Reservoir in Gahwar Field. SPE Middle East Oil Show, Manama, 17-20 March 2001, SPE-68194-MS.

[18] Farrelly, M. and Rabia, H. (1987) Bit Performance and Selection: A Novel Approach. SPE/IADC Drilling Conference, New Orleans, 15-18 March 1987, SPE-16163-MS. https://doi.org/10.2118/16163-MS

[19] Gavito, D.G. (1996) A New Rock Strength Model and Its Practical Applications. International Petroleum Conference and Exhibition of Mexico, Villahermosa, 5-7 March 1996, SPE-35322-MS. 